\title{
Chiptuning Intellectual Property: Digital Culture Between Creative Commons and Moral Economy
}

\author{
Martin J. Zeilinger \\ York University, Canada \\ martin.j.zeilinger@gmail.com
}

\begin{abstract}
This essay considers how chipmusic, a fairly recent form of alternative electronic music, deals with the impact of contemporary intellectual property regimes on creative practices. I survey chipmusicians' reusing of technology and content invoking the era of 8-bit video games, and highlight points of contention between critical perspectives embodied in this art form and intellectual property policy. Exploring current chipmusic dissemination strategies, I contrast the art form's links to appropriation-based creative techniques and the 'demoscene' amateur hacking culture of the 1980s with the chiptune community's currently prevailing reliance on Creative Commons licenses for regulating access. Questioning whether consideration of this alternative licensing scheme can adequately describe shared cultural norms and values that motivate chiptune practices, I conclude by offering the concept of a moral economy of appropriation-based creative techniques as a new framework for understanding digital creative practices that resist conventional intellectual property policy both in form and in content.
\end{abstract}

Keywords: Chipmusic, Creative Commons, Moral Economy, Intellectual Property, Demoscene

\section{Introduction}

The chipmusic community, like many other born-digital creative communities, has a rich tradition of embracing and encouraged open access, collaboration, and sharing. It does not like to operate according to the logic of informational capital and the restrictive enclosure movements this logic engenders. The creation of chipmusic, a form of electronic music based on the repurposing of outdated sound chip technology found in video gaming devices and old home computers, centrally involves the reworking of proprietary cultural materials. As part of practicing their craft, chiptune artists frequently invoke concepts of fairness and justice, yet they are not particularly fond of the law. In what may appear as a contradictory stance, chiptune artists tend to attach copyright licenses (albeit alternative ones) to their creations, yet they rarely enforce these licenses legally. Chiptune artists are eager to make their creative expressions freely accessible to the world, but they also want to retain control over the parameters of this access, and be able to prevent certain uses. This essay is an attempt to navigate the seeming contradictions spelled out above. It provides a new approach to understanding a digital creative community that resists the economic logic of conventional intellectual property law while establishing its own, moral economy of fair conduct in which collective, socio-cultural benefit trumps individual profit.

Three years ago, after many months of production time and achieving its fundraising goal on Kickstarter.com within just four hours, the American blogger and digital entrepreneur Andy Baio released Kind of Bloop, a full song-by-song, note-by-note chipmusic remake of Miles Davis's famous 1959 record Kind of Blue. ${ }^{1}$ True to the 
ideals of the chiptune community, the release was envisioned as a not-for-profit project, with some compensation paid to the five collaborating chiptune musicians. ${ }^{2}$ Because creating chipmusic frequently involves the reusing of proprietary technology as well as existing sound effects and sounds, the album's release had been preceded by discussion concerning copyright issues the project might run into (see Baio 2009a and 2011). Almost universally, chiptune artists favor non-commercial, open access creation and dissemination strategies for their work. However, Baio chose to obtain conventional copyright licenses for the use of the original works in questions (Baio $2009 \mathrm{~b})$. As a result, the community's concerns regarding copyright issues proved unfounded for the main, musical component of the Kind of Bloop project. But then, the creator of a photograph used as a cover for the original release of Miles Davis' Kind of Blue objected to Baio's reusing of the image in a heavily pixelated version as cover art for the chipmusic remake, and threatened to sue for copyright infringement. The open, collaborative, and non-commercial nature of Kind of Bloop could not dissuade the photographer, and Baio, facing statutory fines potentially in the hundreds of thousands of dollars, removed the image and settled out of court for the still-considerable amount of $\$ 32,500$. The controversy sparked widespread debate on high profile blogs and in the chiptune community, which perceived the legal threats as yet another greedmotivated attempt to impede fair use practices and hamper non-commercial, open, and collaborative digital creative efforts. That Baio was threatened with litigation for a very peripheral aspect of the Kind of Bloop project also obscures the important detail that Baio's handling of the main, musical aspect - namely to obtain conventional copyright licenses for reusing Miles Davis' music - is at odds with the practices and ideals evident in the majority of work produced by the chiptune community.

Without doubt, the chipmusic remake of Miles Davis' music is the central aspect of Kind of Bloop. Andy Baio's choice to obtain conventional copyright licenses for reusing Miles Davis' work is unusual in comparison to the larger chiptune community's customary preference, which is to engage existing works with a fair use approach, and to attach Creative Commons licenses to its own creative output. This preference exemplifies the shared values of free, open access to pre-existing as well as new content that members of the chiptune community commonly uphold. Although never formalized, these values tend to guide most chiptune artists' craft. As I will show, they are followed fairly strictly, and are enforced socially, that is, through communication and negotiation commonly occurring in online forums. In practical terms, Baio's decision to obtain licenses for Miles Davis's works was probably correct, given his negative experience with the owner of the reused photograph. Conceptually, however, his decision contradicts most chiptune musicians' perspective on the permissibility of their practice. In fact, Baio himself has argued extensively, on moral as well as on legal grounds, that he considers Kind of Bloop a fair use project (Baio 2011). This marked discrepancy between Baio's legal course of action (to obtain licenses and to settle) and his moral conviction (that the project should be considered fair use) reflects very well the pervasive fear of litigation that grips most digital creative communities today. This fear speaks to the "disempowering structure of a 'copyright wars' way of thinking about how to use the culture at your fingertips" (Aufderheide and Jaszi 2011: xii), and frequently backs creative practitioners into overly cautious legal decisions with which they disagree on ideological terms.

Kind of Bloop exemplifies some of the struggles contemporary digital creators face, in that the project brings together three approaches that appear contradictory: Baio obtained commercial licenses; he allegedly infringed a copyright and settled the ensuing legal dispute out of court; he convincingly argued that the overall project constitutes a fair use of copyright-protected materials. In total, this is indicative of a strong rift between creative communities that hold ideals of free access and noncommercial distribution, and a litigious copyright culture of limited access rights that is backed by powerful lobbies for the ever more restrictive enclosure of intellectual 
property. However, given the manifold restrictions and limitations imposed on digital creators by current intellectual property regimes, I question that Baio's handling of the Kind of Bloop case can really be interpreted as inconsistent. Ultimately, I will argue that his actions were not contradictory at all - they appear so only when they are approached through a discourse of the economically informed, property-based copyright models that dominate our cultural landscape.

After introducing and broadly defining chipmusic, I will develop this argument by considering the chipmusic community's heavy reliance on the Creative Commons licensing model, which has sustained some criticism for being uneasily balanced between ideals of open, free access, on the one hand, and submission to a marketbased logic of intellectual property exchange, on the other. I will argue that the logic of the Creative Commons model - in some ways a continuation of conventional copyright philosophy by other means - cannot satisfactorily explain how the chipmusic community functions. This is confirmed, for example, when considering chipmusic's links to early hacking culture, in particular to the 'demoscene,' and the chiptune community's tendency, derived from this connection, to enforce ownership claims socially rather than legally. As I will show, the legal theory behind the Creative Commons model does little to help us understand what drives predominantly noncommercial and open creative communities such as the chiptune scene, which fundamentally opposes the economic logic of intellectual property rights.

A different explanatory model is needed - one that sidesteps the economic logic underlying intellectual property law and the so-called alternatives it has sparked. In the second half of this essay, I locate this alternative in the concept of moral economy, popularized by the Marxist social historian E. P. Thompson in the early 1970s. Traditionally, political scientists and social historians have used this concept to explain various kinds of informal, seemingly unpredictable resistance levered by pre-market communities against the expansion of a market-based economic logic. The concept has received scant scholarly attention, however, in the context of informational capitalism and digital cultural environments. I propose that the concept of moral economy offers a new approach to questions of why and how digital creators resist assimilation into restrictive intellectual property regimes. In conjunction with my discussion of the creation, dissemination, and roots of chipmusic, the moral economy concept allows me to highlight the discrepancies between the restrictive traditional concept of cultural ownership encoded in contemporary intellectual property law, and the more open and participatory norms of cultural ownership upheld by creative communities such as the chiptune scene. The concept thus proves useful in understanding the cultural and social - rather than economic - logic of expressive forms such as chipmusic.

\section{A Short Chipmusic Primer}

Artists have been creating chipmusic for more than two decades. Nevertheless, perhaps because it is relatively successful in resisting assimilation by the pop music mainstream, the form continues to be characterized as fairly new (see, for example, CBC, 2012). The cognate terms chiptune and chipmusic ${ }^{3}$ broadly describe "the composition and performance of present-day electronic music on obsolete videogame hardware" (Tomczak 2009: 1). Frequently aided by amateur-written software, the music is created through a combination of using existing technology (specifically the sound chips found in old video game consoles and personal computers) and the repurposing of content, such as specific sounds, sound effects, and so on. Intellectual property rights protect much of the digital code reworked and most of the hardware reused in the creation of chipmusic, so that the legal status of the art form is somewhat unclear and remains untested to date. However, due to the relatively low public profile of chiptune artists outside their communities of artists and niche audiences, and the international chiptune scene's overall tendency to produce and disseminate its work 
non-commercially, legal disputes rarely flare up. As will be seen, chiptune artists are able, in fact, to regulate access to and circulation of their work communally and socially (usually in online forums), rather than legally.

Chipmusic boasts very diverse sounds. In a 2003 account in Wired Magazine that drew heavy criticism from the chiptune community, Malcolm McLaren, the selfproclaimed father of punk music, recounted his "discovery" of chipmusic and described it as a new kind of electronic punk, created by "reverse engineering" video game hardware from "the antediluvian 8-bit past" and producing sounds "as though Twiggy were somehow stuck inside Space Invaders" (McLaren 2003: websource). In the same article, chiptune artists were described as "the Velvet Underground of the 21st century" and "the new ABBA". As these disjunctive descriptions suggest, chipmusic cannot be pinned down as a genre. Like punk, it resists dominant takes on form, genre, and cultural ownership. Chiptune artists have experimented with genres ranging from electronic dance music to pop, from jazz to classical music. Chipmusic should be defined as a form rather than a genre, with the sound chip serving as a medium of musical expression. This medium often takes the form of outdated hardware such as the Nintendo Entertainment System and the Nintendo Game Boy, or the Commodore 64 and Atari ST home computers. Chiptune musicians play (that is, program) the sound chips contained in such devices; as noted by the researcher Anders Carlsson, who also creates chiptunes under the pseudonym Goto80, "no matter what is done with a sound chip in terms of harmony, structure or rhythm," the outcome, regardless of an artist's genre preferences, "is still chip music" (Carlsson 2008: 154). Literally, chipmusic refers the repurposing of outdated sound chips for programming compositions that will be generated in real-time whenever the composition code is processed on the chip. In this sense, chiptune compositions bear some similarity to piano rolls, the perforated rolls of paper that were once fed into mechanical pianos, turning them into early, analog music sequencers. ${ }^{4}$

In part because of the appropriative techniques it employs, chipmusic possesses important referential qualities, and can strongly invoke the sounds of specific video game consoles, game genres, or eras of early personal computing and programming. This puts the art form in a contentious relationship with patented sound synthesis technology, with proprietary cultural artifacts such as video game soundtracks, as well as with the production contexts and cultural environments evoked by such artifacts (Zeilinger inpress). Perhaps because of this uncertain relationship to cultural property owned by others, chipmusic, which by now has its regular international public events, annual festivals, dedicated online radio stations, and a growing number of appearances in pop music and mainstream cinema, is difficult to instrumentalize for commercial purposes. It remains, therefore, a site for the experimentation with new creative processes and dissemination strategies that are resolutely poised against the encroachment of conventional intellectual property schemes.

Because of the experimentation, knowledge of the canon, and the relatively high level of technical skill required to create good chiptunes, chipmusic is a very playful art form. Few practitioners enter the scene with ambitions to exploit their craft commercially. Even established chiptune artists foreground the playful aspects of creating chipmusic, which are often shown to the outside world through the nostalgic celebration - in visuals, sounds, and language - of early video game aesthetics. Furthermore, the making of chipmusic is a social activity as much as it is a creative one: virtually all chiptune musicians maintain active profiles on online message boards, many of which also function as open repositories for downloadable music created by members (the largest among these are Chipmusic.org and 8bc.org). ${ }^{5}$ On these websites, discussions of all aspects of production and distribution, performances techniques, and the chipmusic canon parallel the day-to-day interactions of colleagues, friends, and likeminded individuals who share creative interests across different demographics and geographical boundaries. It is also here that the ideals and values 
shared among members of the community are debated and refined, although such discussion never quite amounts to a formalization of the cultural norms that characterize chipmusic and chiptune musicians.

Among the most serious issues discussed on these community websites are the aesthetic integrity of the art form and the moral integrity of the chiptune community itself. As discussed below, heated debate regarding the behavior of musicians who bring discredit to the community can be frequently observed on many discussion threads. These threads predominantly concern the misappropriation of chiptunes in transformative works, most commonly with a focus on plagiarism and the unauthorized commercial exploitation of chiptune artists' work by third parties. It appears, in fact, that in online forums, the chiptune scene rallies and organizes more strongly around this issue than any other. Usually, such debates remain informal and colloquial, yet are carried out on a high level of theoretical sophistication, showing that chipmusic can be all play and serious business at the same time. These debates, as I will show in the last part of this essay, provide an interesting perspective on the moral norms that shape the community, and offer great insights into the inability of conventional intellectual property law to fairly regulate chiptune activities. ${ }^{6}$ In this, they reflect the chiptune community's high regard for fair conduct, openness, collaboration, and sharing.

\section{Excursus: Chipmusic and the Demoscene}

It is helpful to approach chiptune musicians' resistance to attempts of restricting or enclosing their craft through the art form's links to the demoscene, an amateur hacker culture that reached its zenith during the 1980s. The demoscene represents a conceptual precursor for the chiptune scene emerging around the same time, in that both share a philosophy of valuing open access to creative expressions, the sharing of skills and information, and the social as well as technical benefits of collaborative creativity. The 'demos' that gave the demoscene its name resemble short, nonnarrative computer-generated videos. However, this is not an accurate image: rather than being recorded as video, demos are programmed - like chiptunes, their ideal existence is in code. Similar to the real-time generation of a chiptune each time its code is executed, each time a demo is rendered on a screen the time-based audio-visual experience it embodies is generated in real-time by the executable code of which the demo exists. When they first appeared in the late 1970s, demos were attached, as a kind of signature, to computer games whose copy protection had been hacked and removed so that the games could be freely circulated. Such digital, audio-visual signatures identified individual hackers (or groups of hackers), and allowed them to take credit for their work. While demos are today sometimes treated as early iterations of digital video art, many creators of demos still perceive their work primarily as demonstrations of advanced programming skills. ${ }^{7}$

The demoscene maintains a number of creation myths, which include programmingsavvy gamers hacking computer games to remove particularly difficult in-game obstacles, and programmers illegally converting software from NTSC to PAL formats to make them available overseas (see Cox 2009). This range of stories gives a good impression of the values of non-market activities aimed at openness and sharing that characterize the demoscene. In the course of a few years, demos detached themselves from the original purpose of cracking the copy protection of software, and became an expressive form in their own right. The skills they showcased started to go beyond the hacking of digital rights management technology to encompass mastery of visual and musical composition. With many of the basic hardware components of personal computers being almost identical, informal as well as formal competitions among demo writers emerged, further encouraging programmers to explore unintended uses of the available hardware. In this way, demo programmers were pushing the limits of the repurposed hardware and programming environments, and took inspiration from 
the very limitations that defined the hardware at their disposal, in much the same way as many chiptune musicians continue to do.

Now, the challenge became to rend the most impressive amount of audio-visual detail and embellishment from the limited capacities held by early computing devices. This challenge also found, and continues to find, expression in the collaboration between programmers, as well as in the partial reworking of existing demos. The $\mathrm{read} / \mathrm{write}$ formats in which demo files are distributed furthermore means that knowledgeable viewers and users can access original files and modify demos. In fact, as one study of the demoscene points out, many of the visual effects included in demos tend to be "copied from other demos with increased sophistication" (Gruetzmacher 2004: websource). Another notes that this did not result in a larger remix culture, but confirms that the open format of demos made them "suitable for remixing and reverse engineering" (Carlsson 2009: 18). At the time of writing, the online community is aflutter with news that Farbrausch, one of the most widely respected collectives of demo creators, "has released the source codes for most of their projects and tools" (Stamnes 2012: websource), triggering a torrent of open source releases by other demo programmers - a further testament to the demoscene's commitment to values of openness and sharing.

Whether explicitly or implicitly, chiptune artists retain many of the ideals embodied in the demoscene. Today, these ideals manifest themselves partly in the choice of copyright licenses used by chiptune musicians. Most chiptune artists, like creators of demos, furthermore conceptualize their own work as openly accessible, and, when it is transformative in nature, as fair use. As a communicative tool, both demos and chiptunes are participatory, and integrate users on par with creators, within boundaries determined by a shared sense of fairness and justice. And, like demo creators who distributed the results of their experiments freely and showcase their work in read/write formats, many chiptune artists also continue to make their work available in open, $\mathrm{read} / \mathrm{write}$ formats.

\section{The Dissemination of Chipmusic and the Limits of the Creative Commons}

While chiptune musicians continue to uphold these values in theory and practice, several developments have resulted in shifts in how chipmusic is created and disseminated. With easy access to broadband download speeds and powerful processors, and the ubiquity of audio compression algorithms that retain a fair amount of sound quality (such as MP3), adherence to a minimalist programming ethic is no longer a technical requirement for chiptune artists, demo programmers, and other digital creators. As a result, chipmusic is now often created using software that emulates early sound chip architecture, and is circulated in static, read-only audio file formats. The limitations of 8-bit technology can now be embraced as a source of inspiration, rather than of constraint.

The adoption of compressed digital audio files and Internet-based dissemination channels did mean, however, that the chiptune community had to begin paying more attention to how access and dissemination can - and should - be regulated. Based on its conceptual and practical roots, the community favours, as mentioned, open access models. Yet, in a time of the hyper-commodification of many types of cultural expression, unregulated access to cultural expressions can invite the (mis)appropriation of these expressions by profit-oriented third parties. Artists can preempt this unwanted appropriation by claiming conventional copyrights to their works, but many alternative creative communities perceive this as bowing to the same economic logic of cultural exchange that they actually wish to oppose. The increasing commodification and propertization of cultural expressions has led the chiptune community to adopt Creative Commons licenses as one of the most important vehicle to design a balance between control of and free access to chipmusic. Today, the majority of chipmusic labels favored by the community, such as 8bitpeoples.com, use 
Creative Commons licenses. The same is true for most chipmusic-related content posted to websites that allow artists to make their work available for streaming and/or downloading, such as Soundcloud.com and Freemusicarchive.org. Finally, dedicated chipmusic sites such as Chipmusic.org tend to recommend usage of these licenses. At first sight, Creative Commons licenses indeed appear to offer a practicable alternative to conventional copyrights: they are designed to serve creators who are uncomfortable with the rigidity of conventional copyright law, and their flexibility enables creators to specify in detail what kinds of uses they want to allow or prohibit. ${ }^{8}$

Chiptune-related online discussion boards routinely feature active threads on which the use of different Creative Commons licensing schemes is discussed, and on which the apparent benefits of these licenses are explained to newcomers. Receptors, the pseudonymous author of one such thread, recently estimated that "easily $95 \%$ or more is all up as CC" that is, uploaded as Creative Commons-licensed material (Receptors 2012: websource). While this estimate is almost certainly too high for the general chiptune scene, it may approximate an accurate figure for work uploaded to dedicated chipmusic sites. On Chipmusic.org, where this comment was posted, users are expressly advised that "it is a member's responsibility to apply the correct license to the media uploaded to Chipmusic.org and the responsibility of everyone else to respect the licenses attached to the media uploaded to Chipmusic.org" (Chipmusic 2010: websource). No details are provided regarding what specific licenses to use, but it can be assumed that the rule refers to Creative Commons licenses, which are the only type of licenses discussed in depth anywhere on the website, and which are, in fact, by far the most common licenses applied to uploaded work. This kind of implicit recommendation speaks to a tacit agreement, among site moderators and users, with regard to the importance of using alternative licenses to keep chipmusic openly accessible while fending off commercial exploitation of these expressions by third parties. Especially in light of the heavy use of MP3 and other read-only formats for the dissemination of chipmusic, it can be assumed that Creative Commons licenses are seen to serve two important purposes: to protect the community's non-commercial ideals, and to ensure artists' continued ability to rework the creative expressions of their peers. If Creative Commons licenses indeed serve these needs, then it would appear that what the chipmusic scene has lost in adopting new, static file formats for distributing its music, it has made up for by using a licensing system that conforms to the community's ethics of non-commerciality, collaboration, and sharing.

On closer inspection, however, it becomes clear that the Creative Commons licensing system may be less than ideal for the chipmusic community, both in practical and in theoretical terms. Chiptune artists seek protection of their creative expressions primarily so as to better be able to define parameters of relatively unrestricted user access. Creative Commons licenses indeed allow creators to define such parameters. However, they do so by adhering to the underlying logic of conventional copyright licenses - they enable rights holders to "condition their long and strong copyright [and] use the strength of owners' rights to allow owners the leeway to release their works" (Aufderheide and Jaszi 2011: 12). Rather than representing a fundamentally different system of access and rights regulation, Creative Commons licenses simply modify and reframe the ideology of conventional copyrights.

The subject of ongoing debate, the Creative Commons licensing model has not only been celebrated as an alternative to conventional copyright licences, but also as a new viable pathway for independent creators to commercially exploit their works (Foong 2010). Unsurprisingly, this aspect of the Creative Commons model has also drawn criticism, as it problematizes the positioning of the licenses as an alternative to, rather than a variation of conventional copyright licenses (see, for example, Berry and Moss 2005). Conceptually, the Creative Commons licensing model is thus at odds with the values held in the chipmusic community: the licenses remain modeled on the economic logic of conventional copyright law - a logic which chiptune artists, like many other 
creators of digital art, oppose. A further problem is that the ideals of openness and sharing encoded in Creative Commons licenses are enforceable only through conventional legal tools, and in traditional courts of law that otherwise abide by the property-based, economically informed copyright model. For digital creators who wish to distance themselves from these legal and economic models of controlling creative expressions, such a system may be untenable. Ultimately, these enforceability issues also pose practical problems. Since the cost of legal action is generally prohibitive, assurances as to the licenses' enforceability in courts are essentially moot. Not only will many independent creators not want to pursue legal action, most would simply not be able to afford it.

Members of the chiptune community frequently experience problems with the effectiveness of Creative Commons licenses first hand. Among the most high profile cases of the misappropriation of Creative Commons-licensed chipmusic are the illegitimate activities of the internationally successful music acts Crystal Castles and Timbaland (see Gilmore 2012). In the case involving Timbaland, the chiptune artists whose rights were violated chose to litigate, but this remains an isolated example. ${ }^{9}$ For the most part, chiptune artists do not take recourse to traditional legal tools. Instead of taking violations of the rules spelled out in Creative Commons licenses and in the moral code of the chiptune community to court, they seek justice in alternative venues, more specifically in the para-legal spaces of online discussion forums and the comment sections of blogs (Zeilinger 2011). It is here that violators are exposed, ridiculed, and, not infrequently, subjected to prank-based retaliation. In tone, this social enforcement of informal rules and values can range from rational to humorous and outright humiliating. The transgressions in question are commonly debated in a highly sophisticated fashion. Accusations are always based on the presentation of evidence, which is frequently unearthed collectively or by uninvolved parties. ${ }^{10} \mathrm{~A}$ recent example of this kind of controversy is the ousting of the British performer Emma Lundy aka Once We Were Robots, a repeat offender with a moderate amount of commercial success, who was shown to compose much of her music by illicitly sampling freely available work created by active members of the chiptune community (Je Mapelle 2011). A further example is the recent discovery of a Russia-based website (ironically it is called Legalsounds.com) that sells compilations and albums of freely available chipmusic (VCMG 2012).

These actions unfold exclusively online - in discussion threads, blog posts, email correspondences, and denunciatory posts on Facebook and Myspace webpages. The contents and tone of these postings make clear that the chiptune community takes great offense to the violation of the moral rights of the original creators and of the ideals by which the community defines itself. Members of the chiptune community can frequently be seen to vent frustration at the impracticability of Creative Commons licenses. In a comment on the plagiarism committed by Crystal Castles, the chiptune artist M-.-n, for example, lamented the lack of respect for the licences, stating, "It's quite a big deal since a LOT of artists are trusting creative commons and this story puts the license to doubt, since it seems people can break it" (Kirn 2008a: websource).

Other comments show that doubts regarding the effectiveness of the Creative Commons licenses have led many in the chipmusic community to the realization that the economically informed logic underlying copyright law is generally unsuitable for the task of preserving the moral integrity of chipmusic. As one commenter astutely observed, debates around the misappropriation of chipmusic are not "about the law" but rather about violating "a very nebulous code" of a different kind" (Kirn 2008b: websource). This code consists in the shared values and norms of the chiptune community - it is a moral code that Creative Commons licenses cannot enforce or protect. Legal enforcement of this moral code would inevitably have to fall back on the economic logic of conventional copyright law, which runs counter to many ideals held by the chiptune community. For resistance to violations of community norms to be 
perceived as successful and aligned with the community's values, it must take the form of the social enforcement described above.

Put differently, an economically informed policy model such as the Creative Commons cannot successfully regulate the chiptune community's system of the production and exchange of cultural works. Because such a model would force chiptune artists back within the purview of economic considerations, it can also not serve to explain what drives and organizes this community. This raises an important question. Given that the chiptune scene resists - sometimes explicitly, sometimes implicitly - assimilation into a market-based system of commodity exchange, while at the same time heavily relying on a licensing model that is part of this system, what might be an appropriate theoretical framework to explain what motivates and structures the art form? How can the chiptune community's positive stance on appropriation and sharing, which finds expression in artists' creative practice, be reconciled with the community's frequent invocation of ownership concepts?

\section{A Moral Economy of Chipmusic}

I began this article by noting that the chiptune community is characterized by a series of seeming contradictions. Several of these contradictions are brought together in the example of the Kind of Bloop project, whose producer tried to adhere to conventional copyright laws, but who also had to settle a suit for alleged copyright infringement, all the while arguing for the fair use status of his project. We can add to this list the fact that chipmusic practice generally contradicts conventional intellectual property while, at the same time, using (modified) copyright licences. After the foregoing discussion, the argument can now be made that these actions are less contradictory than they initially appear. What they indicate is not so much inconsistency, but rather the inability of market-based, profit-oriented regulation systems to accommodate the ideals, motivations, values, and needs according to which chipmusic is conceptualized. This cluster of norms constitutes the moral economy of chipmusic.

As noted, chiptune artists, like many other digital creators, navigate complex ideas regarding the permissibility of repurposing, copying, and circulating information and cultural works. Their practice embodies principles of open access, collaboration, and sharing, and they have developed strong views on certain activities that they feel are wrong and unethical. These creators have a highly developed sense of fairness, but are neither fond of the legal models our society uses to keep things fair, nor of the economic logic that informs these models. Violations of the perceived rights of chiptune artists, I have argued, are rarely litigated, but para-legal action is nonetheless always brought against them. Some practices, such as the use of Creative Commons licences, inevitably fall within the purview of conventional intellectual property rights; yet the community simultaneously cultivates a sense of operating outside the established logic of the market-based, profit-driven cultural spheres intellectual rights policy institutes. The concept of moral economy aims directly at explaining this seeming contradiction. Without direct recourse to an economic rationale, it can help describe what drives chipmusic practically, aesthetically and ideologically.

In political science and political philosophy, the concept of moral economy is invoked to explore the dynamic, oppositional relationship between shared cultural and moral values and economic activities that interfere with these values. We can observe exactly this kind of relationship in the interplay between the chiptune community and the legal and economic culture it resists. The concept of moral economy was popularized by the British Marxist social historian E. P. Thompson, who published, in the early 1970s, a widely read analysis of the moral and economic logic behind seemingly unpredictable bread riots in eighteenth century England. The goal of these riots was to restore fair access to bread at reasonable prices, yet while these riots appeared to have vaguely economic motivations, Thompson noted that they were 
vehemently poised against the expansion of a modern economic order perceived to contradict traditional community values (Thompson 1971).

The concept of moral economy has been considerably reworked since then, and has been used to analyze resistance staged more recently by other non-market or premarket communities (see, for example, Arnold 2001, for a discussion of water rights, and Banks 2006, for an analysis of community activism by freelance labourers in the creative industries). But Thompson's (1971: 78) original insights still hold: arguing against the apparently "spasmodic" nature of the organized resistance embodied in bread riots and similar direction action taken by the public, and discounting the perception that such action was carried out by random mobs, he described such resistance activities as being motivated by "economic stimuli" yet driven primarily by a shared, communal "legitimizing notion" regarding what is right and wrong, fair and unfair. This observation holds true also for the born-digital activities by way of which the chiptune community resists unfair and unwanted enclosure of its craft by intellectual property regimes. Today, researchers frequently struggle to explain the fluid and seemingly spontaneous formation of digital resistance that ebbs and flows as needed without ever quite constituting itself as formalized groups or organizations. The long tradition of culture jamming, the recent Occupy movement, and the current activities of Anonymous are excellent examples of such resistance. At the moderate end of a spectrum of similar digital-era resistance, we can add the chiptune community and the resistance against expanding intellectual property discourses embodied in its practices.

The successes of digital initiatives pushing for fairness and openness in informational environments are all too often credited to the supposedly innate democratic qualities of digital and networked media environments. While this kind of techno-utopianism is easily exposed as faulty (Benkler 2006 is one good starting point for such a critique), the organising drive behind the organization of digital resistance remains difficult to grasp. I argue with Thompson that, such resistance phenomena do "not require a high degree of organization," but simply "a consensus of support in the community, and an inherited pattern of action with its own objectives and restraints" (1971: 119). In other words, the resistance phenomena in question are driven by shared norms and beliefs, and collective agreement on the importance of holding on to the ideals they embody. Something important is lost, in this sense, when the activities of vibrant creative communities such as the chiptune scene are described only via the instrumental, analytical logic of economic behavior - especially when the community in question vehemently opposes this logic.

The social and political phenomena discussed by Thompson and the characteristics of the chiptune community display important similarities. The riots analysed by Thompson were sparked by hunger, by a fight for control over access to vital means of subsistence. Chiptune artists, by comparison, require control over their and others' fair access to informational goods and technology, rather than affordable access to bread. Clearly, there are important differences between the two. As a means of subsistence, however, bread is not only necessary for survival. The modalities of its availability also strongly impact a society's social fabric. Accordingly, we should understand a moral economy to be concerned not necessarily only with means of subsistence, but with social goods more generally (see Arnold 2001) - a category that certainly accommodates the cultural works at stake in born-digital phenomena such as chipmusic. Bracketing matters of survival and focusing on the social and communal aspects of the moral economy concept, in this sense there is a parallel to be drawn between the vitality of access to food in a pre-industrial era and access to informational goods today. More and more, this parallel is also strengthened by the international recognition of access to information as a fundamental human right.

Moral economies reflect patterns of social resistance that are directed against the encroachment of expanding economic models on common grounds, expansions that are often experienced as unfairly disadvantaging the public (including artists, everyday 
consumers, users, etc.). Thompson saw the "legitimizing notion" that validated such resistance as strong enough to override the economic logic imposed and enforced by the authorities. In other words, the grievances that sparked the riots he studied "operated within a popular consensus as to what were legitimate and what where illegitimate practices" (1971: 78). This consensus, Thompson continues, "was grounded upon a consistent traditional view of social norms and obligations, of the proper economic functions of several parties within the community. ... An outrage to these moral assumptions, quite as much as actual deprivation, was the usual occasion for direct action" (ibid.: 79). In the context of present day, digital-based resistance, this direct action can take the form, for example, of protests organised through social media tools, or the Distributed Denial of Service (DDoS) attacks frequently launched by Anonymous and related groups. But it can also take the less aggressive form of longterm opposition against economically informed intellectual property policy that is perceived as unfair - such as is manifest in digital users' networked informationsharing behavior around the globe (see Svensson and Larsson 2012), and in the chiptune community's use of alternative rights regulation models and its web-based social enforcement of perceived ownership rights as discussed above.

Against this view, it could be argued that such action does not effectively resolve ownership disputes in the way in which, for example, copyright litigation would. But the concept of ownership that is at stake in the misappropriation of chipmusic is not based on private property as it is traditionally understood. In the Timbaland case, for example, many community members chose to retaliate without recourse to property law. In a representative move, a chiptune artist operating under the pseudonym cableglitchtv reappropriated Timbaland's plagiarism by entirely remaking and releasing the (copyrighted) song in question in the Game Boy composition tool LSDJ (see Kopstein 2011). Such activity is not concerned with the misappropriation of private property. Instead, the aim here is to re-establish a sense of propriety, and, perhaps even more importantly, to make sure Timbaland gets owned in the colloquial, cultural sense of being put in one's place. The above-cited extensive debate regarding Emma Lundy's plagiarism and commercial exploitation of other artists' freely accessible chipmusic offers another good example. At no point did this debate turn on the issues normally found in infringement complaints (such as requests for lost royalties, or demands for the destruction of violating materials). Instead, the community satisfied its need to assert cultural ownership by exposing the violations on chipmusic websites as well as social media sites, reporting the violator to her business contacts in industry (such as concert bookers and web-label administrators), posting parody videos mocking Lundy, and debating the practicability of various retaliatory pranks. Ultimately, a balance was felt to have been re-established, and with a number of comments confirming the perception that justice had been served - such as, "ding dong the witch is dead! (I'm happy that things have been accomplished!)" and "This has all just given me a strong urge to go and make some new music" - the discussion thread was closed after two days of frenzied activity (Je Mapelle 2011: websource).

Generally, the riots Thompson describes occurred when official regulation "was drifting apart from reality" (1971: 87) - much like intellectual property regimes are today seen to become detached from the realities of contemporary everyday life and the opportunities for copying-based communicative and creative activities it enables. The bread rioters were not, however, opposed to regulation as a matter of principle. In fact, during the recurrent rioting, there was always "a deeply-felt conviction that prices ought, in times, of dearth, to be regulated, and that the profiteer put himself outside of society" (ibid.: 112). Similarly, chiptune artists do not oppose regulation in principle. Their prevalent use of Creative Commons licences indicates that a certain measure of control is perceived as necessary. However, dominant regulation models are perceived to be out of touch with the needs and values of the community, and what follows is 
collective action that opposes and/or sidesteps the overreach of dominant regulation models.

This is well exemplified by a prominently placed official statement on Chipmusic.org, which immediately follows the reminder, quoted earlier, that it "is a member's responsibility to apply the correct license to the media uploaded". Closely resembling standard copyright-related notices, the statement reads:

Unless a member has been given implicit permission of use by the original material owner ... or has been given permission via the terms of a Creative Commons license or copyright ..., members may not copy, modify, publish, transmit, upload, participate in the transfer or sale of, reproduce, created derivative works based on, distribute, perform, display, or in any other way exploit, any of the content, software, materials or services posted to Chipmusic.org... (Chipmusic 2010: websource).

Implicit permission is always assumed by a user of copyrighted materials; semantically, it cannot be granted by rights holders. Use of the phrase at the beginning of the list of online forum rules thus neutralizes everything that follows. Rather than a call to order, the statement is a sleight of hand that we can read as inciting and authorizing copying activities that would not be accommodated by conventional regulation models - activities which conform to the chiptune community's moral economy, but not necessarily to the broader economic order within which the creative community operates. In this sense, the requirement of an implicit permission to use protected materials represents an encouragement not so much to obtain licenses, but rather to apply a fair use perspective to the reuse of existing materials. The large community of artists using this forum clearly agrees, then, that certain uses ought to be permissible, while others ought not to be. Regulation is required, in other words, yet dominant economically driven regulation models interfere with the chiptune artists' own strongly held views of what is fair and what is unfair, of what is right and what is wrong.

Embodying a form of resistance that favors certain kinds of regulation over others, the activities of the chiptune community, like Thompson's bread riots, do not strive for revolution, but for fairness and reasonableness. This ambition can be assumed to also motivate the first example raised in this essay, namely Andy Baio's handling of the Kind of Bloop incident. I would argue that it applies equally to many other community-run initiatives against the overreach and perceived unfairness that defines much of today's economically motivated intellectual property discourse. When the monetary and social costs of access to information (or to bread, for that matter) become untenable, communities that experience not only the economic but also the social impact of regulation will seek to counter the overall market price of access to a social good - a price that it is influenced, in the informational context, by the expansion of intellectual property regimes, DRM restrictions, length and strength of copyrights, etc. The counter measure, as Thompson pointed out, is a "moral price set by the crowd" (1971: 126). It manifests itself in collective attitudes towards the social goods in question and in the social measures taken to enforce the "pricing," in other words, to regulate access. Most importantly, it is manifest "in the cultural self-understandings of the actors themselves, that is, their sense of propriety, justice, obligation, and the like" (Scott 1976: 3-4).

The moral economy of any community remains impossible to reduce to a short definition. It can only be identified in large clusters of practices that are informed by complex sets of shared values, and by the position of fluid, fleeting groups in relation to the economic orders they oppose. As I hope to have conveyed, many of the values that constitute the moral economy of chipmusic are manifest in chiptunes themselves. A reminder of the parallels between chiptunes and the demoscene shows this: like demos, chiptunes are generally "not commercially produced for sale" like demos, they frequently push the "supposed limits" of the environment in which they are created; for those with "highly specialized knowledge" the resulting works are often accessible for modification and reworking; and lastly, many demos and chiptunes show "evidence of cross national co-operation in production" (Borzyskowski 2000: websource). As noted 
above, these characteristics, in turn, are reflected in musicians' and audiences' attitudes towards their works, as well as in their practices of disseminating them.

What Scott (1976: 3 ) calls the "cultural self-understanding" that constitutes a moral economy is precisely what I have attempted to locate in chipmusic practices throughout this essay, contrasting it with the underlying rationale of the alternative Creative Commons licensing model, which remains tied to the agendas of conventional copyright law. The outline of a moral economy of chipmusic presented here remains basic, and requires further development. But even in its tentative form it demonstrates the utility of this approach in conceptualizing the cultural and social significance of digital practices such as the creation of chipmusic, because it can sidestep the instrumental logic that informs economic approaches as well as alternative approaches that remain tied to the economic. As such, my invocation of the concept of moral economy confirms that this instrumental logic of economics, which lies at the core of contemporary intellectual property discourse, is not only ill-suited to legally accommodate many current, digital-based creative practices (a view that is becoming ever more widely accepted), but equally ill-suited to grasping the ideals that drive such practices.

Thompson himself mused about the applicability of the moral economy concept for more recent phenomena. He identified a continuity in resistant activities from the "bread-nexus" that dominated eighteenth century resistance to the "cash-nexus" that began to characterize capitalist societies after the industrial revolution (1971: 79). To this, I propose, we can add an information-nexus that continues to drive resistance along similar lines. To date, there are no sustained efforts to bring the concept of moral economy to critically bear upon contemporary, post-industrial contexts. Only two short texts - one an academic essay, the other an industry whitepaper - consider, not quite adequately, the moral economies of digital media environments (Austin, Jenkins et al. 2006, Haigh 2010). But even though the moral economy concept has received scant scholarly attention in these contexts, I believe its relevance for the study of digital phenomena that stage various kinds of resistance against enclosure through expanding intellectual property regimes is evident. In any historical moment, and in any economic context, there will be actors who are "self-consciously engaged in forms of practice that contain ideas about what is 'good' (and therefore 'bad'), exhibit moral ways of acting towards others, and negotiate the balance between holding instrumental or non-instrumental values" (Banks 2006: 456). Both offline and online, in analog and in digital contexts, there will always be normative social values poised against the policies and regulation measures that spring from the instrumental reasoning informed by economic agendas. As my analysis of the historical roots, creative practice, and dissemination strategies of chipmusic has shown, these values need not gain legitimacy through an ambition or ability to profit - even though the value of culture (and our desire to own it) is now often determined in this way. Rather, such values can assert their legitimacy through the invocation of shared social, political, and cultural viewpoints, which are staged in non-market-based systems of open, collaborative creativity and communication that amount to powerful alternative moral economies.

\section{Endnotes}

\footnotetext{
${ }^{1}$ Previews of the remakes are available at http://kindofbloop.com. Representative example of chiptunes can be accessed at http://chipmusic.org/music\#f=10.

2 The five chiptune musicians Andy Baio invited to contribute to Kind of Bloop were: Chris $\mathrm{J}$. Hampton (aka AstOr), Rich Vreeland (aka Disasterpeace), Sergio de Prado (aka Sergeeo), Samuel Ascher-Weiss (aka Shnabubula), and Jake Kaufman (aka Virt).

${ }^{3}$ Carlsson (2012) includes a thorough analysis of terms' usage.

${ }^{4}$ I significantly expand on this definition in a forthcoming book chapter (Zeilinger 2013). A detailed discussion of the technical aspects of chipmusic creation, including discussion of
} 
various production platforms and their precursors, is available in Driscoll and Diaz (2009). One of several relatively comprehensive history of chipmusic was recently published by Thomas Gilmore (2012), who also creates chipmusic under the pseudonym 10k.

${ }^{5} 8 b c$.org has been experiencing technical problems since early 2012, sparking a migration of members to Chipmusic.org.

${ }^{6}$ My forthcoming book chapter, cited above, contains a detailed analysis of one prominent example of such a debate, concerning the Canadian commercial band Crystal Castles's plagiarism of works by a host of prominent chipmusicians.

${ }^{7}$ A collection of video recordings of accomplished demos is available at http://www.youtube.com/user/TheDemoTube. See Borzyskowski (2000), Hartmann (2010), and Gruetzmacher (2004) for extensive discussion of historical and technical aspects of the demoscene.

${ }^{8}$ The Creative Commons website provides detailed descriptions of the licenses at http://creativecommons.org/about.

${ }^{9}$ The Wikipedia entry on the issue contains references to detailed coverage of the litigation (Wikipedia, 2012).

${ }^{10} \mathrm{See}$, for example, nitro2k01's spectral graph analyses circulated as part of the informal case made against Crystal Castles (nitro2k01, 2008).

\section{Acknowledgements}

I would like to acknowledge the help of my research assistant Dawn Anthony (York University), as well as valuable feedback on a draft version of this essay by Ronald $\mathrm{Ng}$, Sarah O'Brien, and Rachel Freedman at the University of Toronto.

\section{References}

\section{Bibliography}

8Bit Collective. http://8bc.org/ Accessed: 24 Apr 2012.

8bitpeoples. http://www.8bitpeoples.com/ Accessed: 27 Apr 2012.

Arnold, Thomas C (2001) Rethinking Moral Economy. American Political Science Review 95 (1): 85-95.

Aufderheide, Pat \& Jaszi, Peter (2011) Reclaiming Fair Use: How to Put Balance Back in Copyright. Chicago: University of Chicago Press.

Austin, Alec, Henry Jenkins et al. (2006) How to Turn Pirates Into Loyalists: The Moral Economy and an Alternative Response to File Sharing. www.convergenceculture.org Accessed: 24 Apr 2012.

Baio, Andy -

(2009a) Kind of Bloop: An 8-Bit Tribute to Miles Davis. Kickstarter. http://www.kickstarter.com/projects/waxpancake/kind-of-bloop-an-8-bit-tribute-tomiles-davis Accessed: 25 Apr 2012.

(2009b) Kind of Bloop: An 8-Bit Tribute to Miles Davis. http://waxy.org/2009/05/kind of bloop/ Accessed: 3 May 2012. (2011) Kind of Screwed. http://waxy.org/2011/06/kind of screwed/ Accessed: 24 Apr 2012.

Banks, Mark (2006) Moral Economy and Cultural Work. Sociology 40 (3): 455-472.

Benkler, Yochai (2006) The Wealth of Networks: How Social Production Transforms Markets and Freedom. New Haven: Yale University Press.

Berry, David M. \& Moss, Giles (2005) On the 'Creative Commons': a Critique of the Commons Without Commonalty. Free Software Magazine 5: 1-4.

Borzyskowski, George (2000) The Hacker Demo Scene and Its Cultural Artifacts. http://www.scheib.net/play/demos/what/borzyskowski/ Accessed: 6 Apr 2012. 
Carlsson, Anders -

(2008) Chip Music: Low-Tech Data Music Sharing. In Karen Collins Ed. From PacMan to Pop Music. Farnham: Ashgate Press: 153-162.

(2009) The Forgotten Pioneers of Creative Hacking and Social Networking Introducing the Demoscene. Re:live Media Histories Conference Proceedings. University of Melbourne \& Victorian College for the Arts and Music 2009: 16-20. (2012) What Happened in 2006? http://chipflip.wordpress.com/2012/03/16/whathappened-in-2006/ Accessed: 27 Apr 2012.

CBC.ca (2012) Super Mario at a Rave! http://www.cbc.ca/cinqasix/music/2012/01/28/super-mario-at-a-rave/ Accessed: 6 Apr 2012.

Chipmusic.org http://chipmusic.org/ Accessed: 23 Apr 2012.

Chipmusic.org (2010) Forum Rules. http://chipmusic.org/forums/topic/21/forum-rules/ Accessed: 8 Apr 2012.

Cox, Alex (2009) Past, Present and Future of the Demoscene. Techradar.com, 19 July. http://www.techradar.com/news/world-of-tech/past-present-and-future-of-thedemoscene-616817 Accessed: 6 Apr 2012.

Driscoll, Kevin \& Diaz, Joshua (2009) Endless loop: A brief history of chiptunes. Transformative Works and Cultures 2. http://journal.transformativeworks.org/index.php/twc/article/view/96/94 Accessed: 27 Apr 2012.

Foong, Cheryl (2010) Sharing with Creative Commons: a Business Model for Content Creators. Platform: Journal of Media and Communication (December): 64-93.

Free Music Archive. http://freemusicarchive.org/ Accessed: 27 Apr 2012.

Gilmore, Thomas (2012) A Brief History of Chipmusic. The Music Network, 27 February. http://www.themusicnetwork.com/music-features/industry/2012/02/27/abrief-history-of-chipmusic/ Accessed: 7 Apr 2012.

Gruetzmacher, Thomas (2004) PC Demoscene FAQ. http://tomaes.32x.de/text/pcdemoscene faq.txt Accessed: 6 Apr 2012.

Haigh, Maria (2010) Of Ducks and Downloads: The Moral Economy of Intellectual Property in Post-Soviet Society. Libri 59 (4): 248-258.

Hartmann, Doreen (2010) Computer Demos and the Demoscene: Artistic Subcultural Innovation in Real-Time. Dortmund/Germany: ISEA 2010 Ruhr Conference Proceedings: 124-126.

Je Mapelle (2011) This Girl Is Stealing chipt00ns. http://chipmusic.org/forums/topic/4272/this-girl-is-stealing-chipt00ns/ Accessed: 11 Apr 2012.

Kirn, Peter-

(2008a) Chiptune Music Theft Continues; Crystal Castles Abuses Creative Commons License. Create Digital Music, 5 May.

http://createdigitalmusic.com/2008/05/05/chiptune-music-theft-continues-crystalcastles-abuses-creative-commons-license/ Accessed: 29 Sep 2009.

(2008b) CSI: Chiptune - nitro2k01 Gets Scientific with Alleged Violations; Crystal Castles Responds. Create Digital Music, 6 May.

http://createdigitalmusic.com/2008/05/06/csi-chiptune-nitro2k01-gets-scientific-withalleged-violations-crystal-castles-responds/\#more-3418 Accessed: 29 Sep 2009.

Kopstein, Joshua (2011) Timbaland Stole Chiptune, The Internet Stole It Back. http://motherboard.vice.com/2011/3/18/timbaland-stole-chiptune-the-internet-stole-itback--2 Accessed: 2 May 2012. 
Legalsounds. <http://www.legalsounds.com/> consulted: 23 Apr 2012.

McLaren, Malcolm (2003) 8-Bit Punk. Wired.

http://www.wired.com/wired/archive/11.11/mclaren pr.html Accessed: 7 Oct 2009.

nitro2k01 (2008) Crystal Castles and Chip Music Copyright Infringements.

http://blog.gg8.se/wordpress/2008/05/06/crystal-castles-and-chip-music-copyrightinfringements/ Accessed: 12 Apr 2010.

Receptors (2012) Developing Pitfalls of Creative Commons Licensing? http://chipmusic.org/forums/topic/5886/developing-pitfalls-of-creative-commonslicensing/ Accessed: 8 Apr 2012.

Scott, James C. (1976) The Moral Economy of the Peasant. New Haven: Yale University Press.

SoundCloud.com. http://soundcloud.com/ Accessed: 28 Apr 2012.

Stamnes, Bent (2012) The Great Demoscene Sourcecode Giveaway. http://www.displayhack.org/2012/the-great-demoscene-sourcecode-giveaway/ Accessed: 2 May 2012.

Svensson, Mans \& Larsson, Stefan (2012) Intellectual property law compliance in Europe: Illegal file sharing and the role of social norms. New Media \& Society, Prepublished 10 Apr 2012. doi:10.1177/1461444812439553.

Thompson, E. P. (1971) The Moral Economy of the English Crowd in the Eighteenth Century. Past \& Present 50: 76-136.

Tomczak, Sebastian (2009) Authenticity and Emulation: Chiptune in the Early TwentyFirst Century. International Computer Music Conference Proceedings, Queens University Belfast: n.p.

VCMG (2012) LegalSounds is selling YOUR music for their profit! http://chipmusic.org/forums/topic/5943/legalsounds-is-selling-your-music-for-theirprofit Accessed: 11 Apr 2012.

Wikipedia. Timbaland plagiarism controversy. http://en.wikipedia.org/wiki/Timbaland plagiarism controversy Accessed: 2 May 2012.

Zeilinger, Martin -

(2011) Remix Cultures and the Imagining of Alternative Intellectual Property Policy. ISEA 2011 Conference Proceedings, Sabanci University, Istanbul/Turkey. http://isea2011.sabanciuniv.edu/paper/remix-cultures-and-imagining-alternativeintellectual-property-policies Accessed: 2 May 2012.

(2013) Chipmusic, Out of Tune: Crystal Castles and the Misappropriation of Licensed Sound. In Rosemary J. Coombe, Darren Wershler \& Martin Zeilinger Eds. Dynamic Fair Dealing: Creating Canadian Culture Online. Toronto: University of Toronto Press. 